\title{
Status Epilepticus
}

\author{
G. Hagemann ${ }^{*}, 1,2$ J. Zinke ${ }^{2}$ and T.J. von Oertzen ${ }^{3}$ \\ ${ }^{1}$ HELIOS Klinik Berlin-Buch, Dept. of Neurology, Berlin, Germany, \\ ${ }^{2}$ Hans Berger Clinic for Neurology, University Hospital Jena, Germany \\ ${ }^{3}$ Epilepsy Group, Atkinson Morley Neuroscience Centre, St. George's Hospital, London, UK
}

\begin{abstract}
Generalized convulsive status epilepticus (GCSE) is a neurological emergency with high mortality which needs immediate recognition and treatment. There is a variety of different categories of status epilepticus (SE) and the form and aetiology of status are important predictors for prognosis. As early treatment is beneficial out-of-hospital treatment can be advised. The first-line treatment of GCSE is lorazepam which can be followed by phenytoin and induction of coma by anaesthetics. Because of receptor changes short-term prognosis to stop seizures becomes worse with time and the longterm prognosis is worst in case of refractory SE. Non convulsive forms of SE can only be diagnosed with an EEG and should be treated more cautiously to prevent potentially harmful adverse events of therapy. Some of the modern anticonvulsants which are available for intravenous use may have good efficacy and tolerability but are not licensed for use in SE, yet. Therefore, they should only be used with neurology specialist advise. For all institutions it is advisable to consent on an in-house protocol which all physicians are familiar with. This review gives a pragmatic overview of diagnosis, classification, and treatment of SE.
\end{abstract}

Keywords: Convulsive status epilepticus, burst suppression, non-convulsive status epilepticus, refractory status epilepticus.

\section{INTRODUCTION}

Status epilepticus (SE) is a probably underdiagnosed condition, which may be life-threatening. The generalized convulsive status epilepticus (GCSE) is characterized by high mortality and morbidity, with an incidence estimated at about 10-20 per 100,000 in Central Europe per year [1,2]. While single seizures are usually self limiting, in SE there is either long lasting continuous EEG or clinical seizure activity or repeated seizures within a 30 minutes time period without regaining consciousness [3, 4]. Recently, this definition has been modified for practical reasons and now seizure activity, particularly in generalized tonic-clonic SE, persisting for more than 5 minutes is addressed as SE. This takes into account the necessity to start treatment early as this improves the chance to stop the status as well as to minimize long-term sequelae $[5,6]$. While the recognition of the typical convulsive status epilepticus is straightforward there are a variety of subgroups which have to be considered when dealing with either confused, somnolent, or comatose patients $[7,8]$. This has also to be considered when dealing with patients with an alteration of consciousness in the hospital especially on the ICU (intensive care unit). The aim of this review is to overview the current knowledge of SE with special emphasis on diagnosis and treatment options.

\section{EPIDEMIOLOGY AND CLASSIFICATION}

The annual incidence rate of SE varies and was estimated to be around 20 per 100000 with the highest incidences in

*Address correspondence to this author at the HELIOS Klinik Berlin-Buch, Dept. of Neurology, Schwanebecker Chaussee 50, D-13125 Berlin, Germany; Tel: +4930940154200; Fax: +4930940154209;

E-mail: georg.hagemann@helios-kliniken.de children and the elderly $[2,6,9,10]$. Variations in figures can partly be explained by status-type specific incidencerates and furthermore, by difficulties in classification per se (Table 1). Although the generalized convulsive status is by far the most critical form of SE there are in fact as many forms of $\mathrm{SE}$ as there are seizure types. Generalized convulsive SE in adults has an incidence of 3,6 to 6,6 per 100000 and a short-term mortality rate around 20 per cent which may even be higher in resource poor countries. Naturally the prognosis is vastly influenced by seizure type, aetiology and duration of SE and age of the patient [11, 12]. One difficulty in classification is the lack of clear definitions and the use of different classification schemes at a time. It has been proposed to use different axes of classification, e.g. in a first step determine clinically whether the SE is convulsive or non-convulsive and in a second step to decide on additional electrophysiological basis or medical history whether the SE is focal or generalized (Table 2) [10]. For further subclassification according to the International League Against Epilepsy additional information is needed [13].

Table 1. Proposed Definitions

\begin{tabular}{|c|}
\hline Status epilepticus (SE): \\
\hline - Generalized tonic-clonic seizure longer than $5 \mathrm{~min}$ \\
\hline - Focal seizures or absences lasting longer than 20-30 min * \\
\hline $\begin{array}{l}\text { - Series of seizures of same duration without full clinical or } \\
\text { electroencephalographic recovery }\end{array}$ \\
\hline Refractory status epilepticus (RSE): \\
\hline $\begin{array}{l}\text { - a status that does not respond to initial anticonvulsant treatment with } \\
\text { first line drugs }{ }^{* *}\end{array}$ \\
\hline
\end{tabular}


Table 2. Classification Scheme of Status Epilepticus

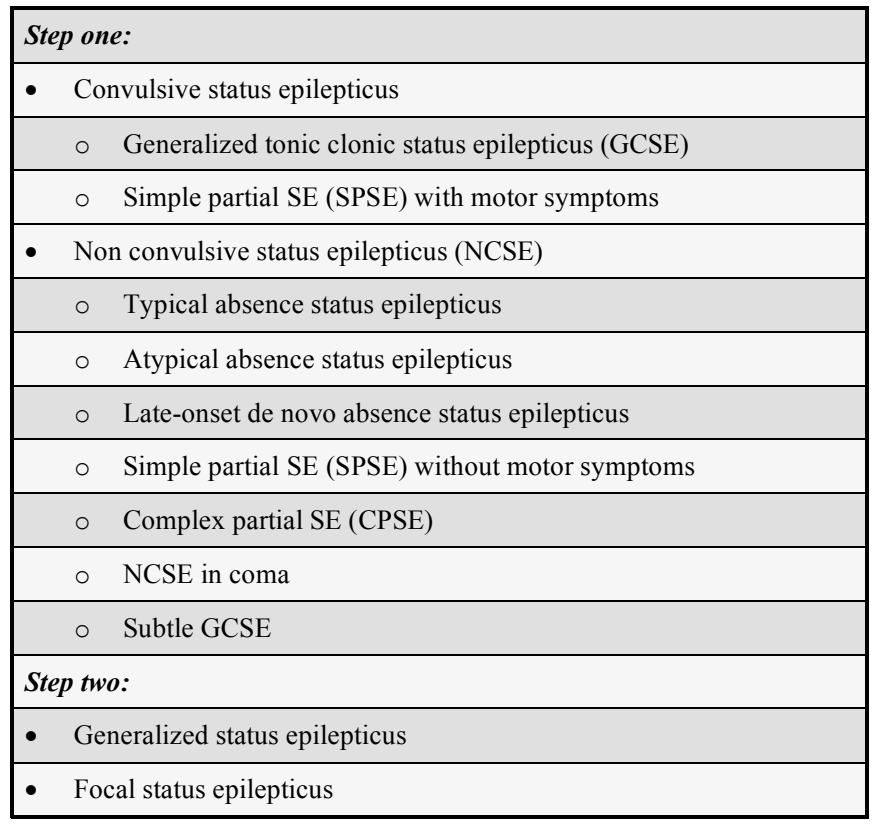

From a pragmatic point of view it might be sufficient to concentrate on categories which make a difference with respect to either treatment options or prognoses. The most important and easy to diagnose category is a generalized tonic clonic status. This is an absolute emergency situation and needs instantaneous treatment. In all other situations less aggressive treatment can be initiated after careful consideration and with some delay. The diagnosis of simple partial SE is likewise straightforward in case of motor symptoms but can be difficult if only subjective e.g. sensory symptoms predominate. Non convulsive status epilepticus (NCSE) is a subtype of SE without any overt motor signs. There is no universal definition to date and NCSE comprises a variety of temporary changes in behaviour or mental status usually associated with continuous epileptiform discharges in the electroencephalogram [8]. It can occur in formerly healthy subjects, in patients with known epilepsy, and in patients hospitalized because of other reasons without a former history of epilepsy (Fig. 1). The latter group may be the most difficult to diagnose especially in ICU patients [1416].

The term refractory status epilepticus (RSE) is used for persistent SE despite adequate initial treatment with a firstline anticonvulsant drug $[12,17]$. There is uncertainty whether the duration of the status has to be considered (Table 1). Refractory SE (RSE) has the worst prognosis with longer seizure duration, longer stay in hospital and the ICU, higher probability to develop epilepsy later on, lower probability to return to baseline clinical level, and mortality rates 2 to 5 fold higher than in non-RSE [12, 17-19].

Having the possibility to use continuous EEG on the ICU in some patients epileptiform electrographic activity persists after therapy of either SE or refractory SE. The significance of a variety of different EEG pattern which can evolve, called ictal to interictal continuum, is not yet clear [20] but usually is accompanied by a poor prognosis. In this category belong periodic lateralized epileptiform discharges (PLEDS), which, despite the name do not point towards clinical seizures or epilepsy and are highly refractory to anticonvulsant medication (Fig. 2). Sometimes the term "subtle status" is used in the situation when ictal EEG activity persists despite therapy. This term likewise, is not well defined and disputable [10, 21-23].

Absence status epilepticus may present either as "typical" or "atypical" status, both demonstrating regular spike-wave discharges with variable morphology and frequency. Lateonset de novo absence status epilepticus is an important differential diagnosis in patients with alteration in consciousness or behavioural changes [10, 24]. It can occur in known but remitted idiopathic generalized epilepsy or as a true de novo manifestation of epilepsy and therefore can be difficult to diagnose (Fig. 3).

\section{DIAGNOSTIC WORK-UP}

While in children the most common cause for SE is febrile seizures or infections with fever, in adults ischemic cerebrovascular disease and hemorrhages predominate [6, $25,26]$. In both age groups a significant proportion of SE are caused by drug withdrawal, compliance problems, or inadequate drug levels. Therefore it is important to differentiate between SE in patients with known epilepsy and in patients with de novo epilepsy or SE. While in epilepsy patients control of AED blood levels and prompt re-initiation of the formerly used anticonvulsive therapy might be sufficient, de novo SE need a more thorough evaluation of causative factors. In fact, there are a variety of differential diagnoses which have to be considered early and before potentially harmful, aggressive treatment is initiated. In any case glucose level, serum electrolytes, blood count, liver and kidney function tests, and a drug screen for therapeutic and toxic substances should be taken. In parallel a focussed neurological examination must be performed and special care must be taken to avoid misdiagnosing a psychiatric disease, particularly non-epileptic SE. In adults and in children early imaging can be recommended to evaluate the aetiology of SE and to direct therapy [26, 27]. While in NCSE the electroencephalogram is of utmost importance, it may be difficult to record a readable EEG in GCSE [23]. The main goal, once a GCSE is suspected, is to exclude a nonepileptic event, especially in a setting when no experienced clinician or epileptologist is available to make this decision on clinical ground.

\section{GENERAL AND PHARMACOLOGICAL TREAT- MENT OPTIONS}

The first goal of treatment is the stabilisation of cardio respiratory function in terms of airway control, breathing, and circulation and to prevent injuries by removing potentially harmful surroundings. In case of uncertainty about the reasons SE it might be wise to administer glucose and, especially in suspected ethanol associated seizures, thiamine (e.g. $100 \mathrm{mg}$ ) intravenously. Although several guidelines for the management of SE have been published by neurological associations there is no general consensus which treatment should be initiated [25, 28, 29]. For practical reasons it can be recommended to stick to routine procedures and implement own guidelines for the 
(a)

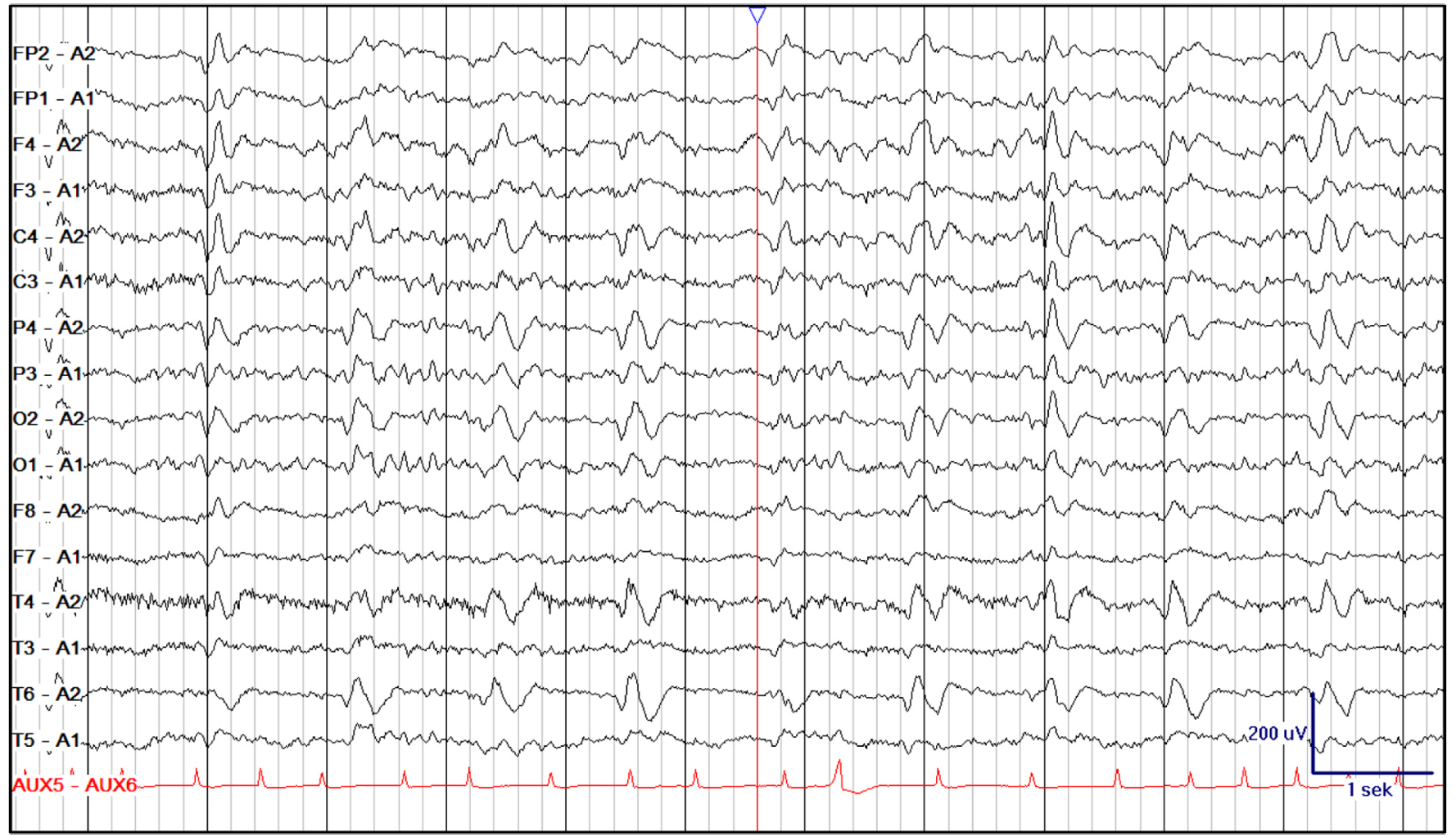

(b)

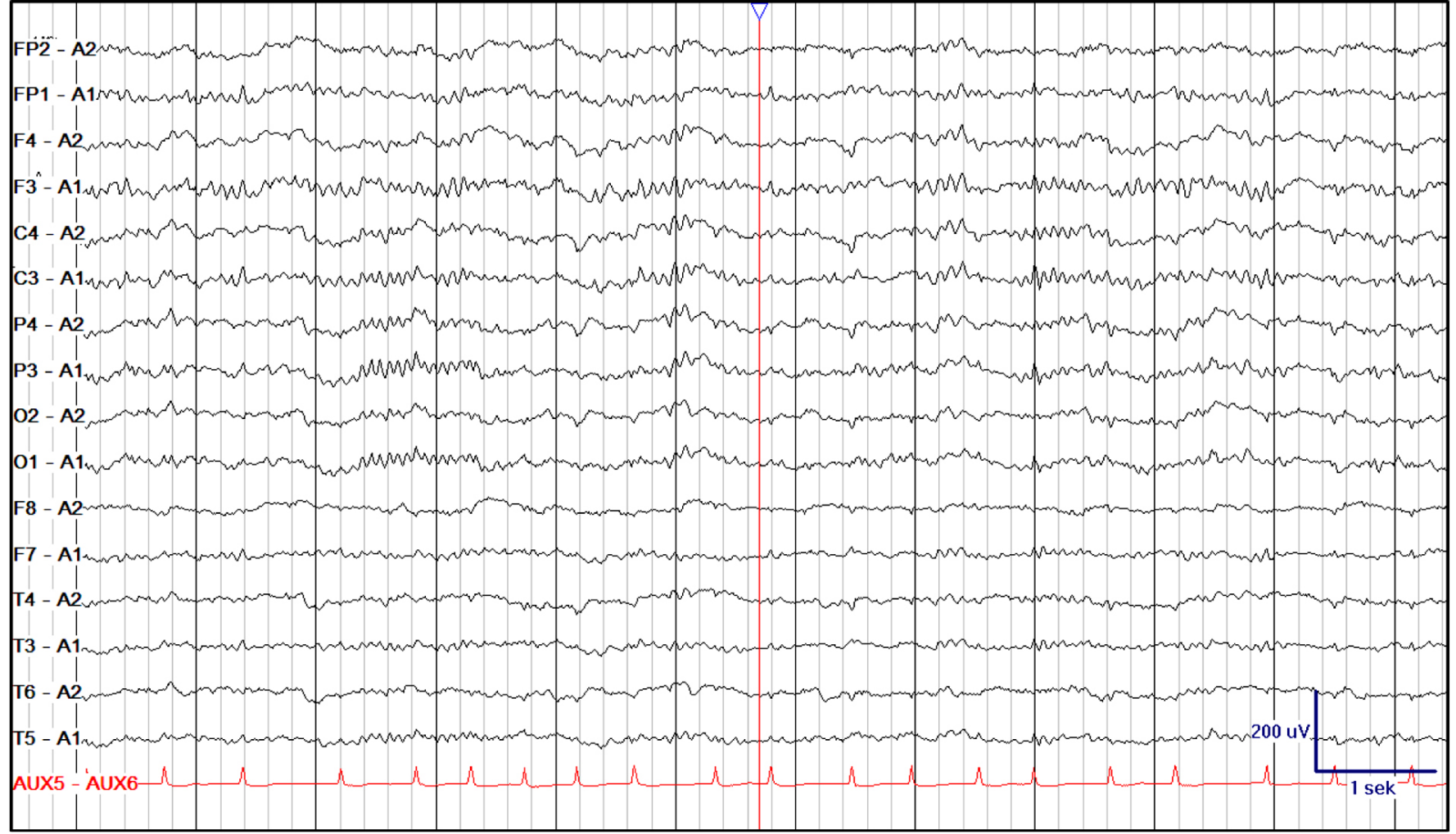

Fig. (1). EEG of a 79 year old female with de novo manifestation of NCSE after right sided cerebral ischemia and intracerebral haemorrhage. Upper row: Note right sided, rhythmic sharp waves with temporal accentuation on a slowed background activity. Lower row: EEG shortly after intravenous infusion of $2 \mathrm{mg}$ clonazepam with a prompt cessation of ictal activity backing the diagnosis of NCSE. Note: clonazepam was given because of timelier availability. 
(a)

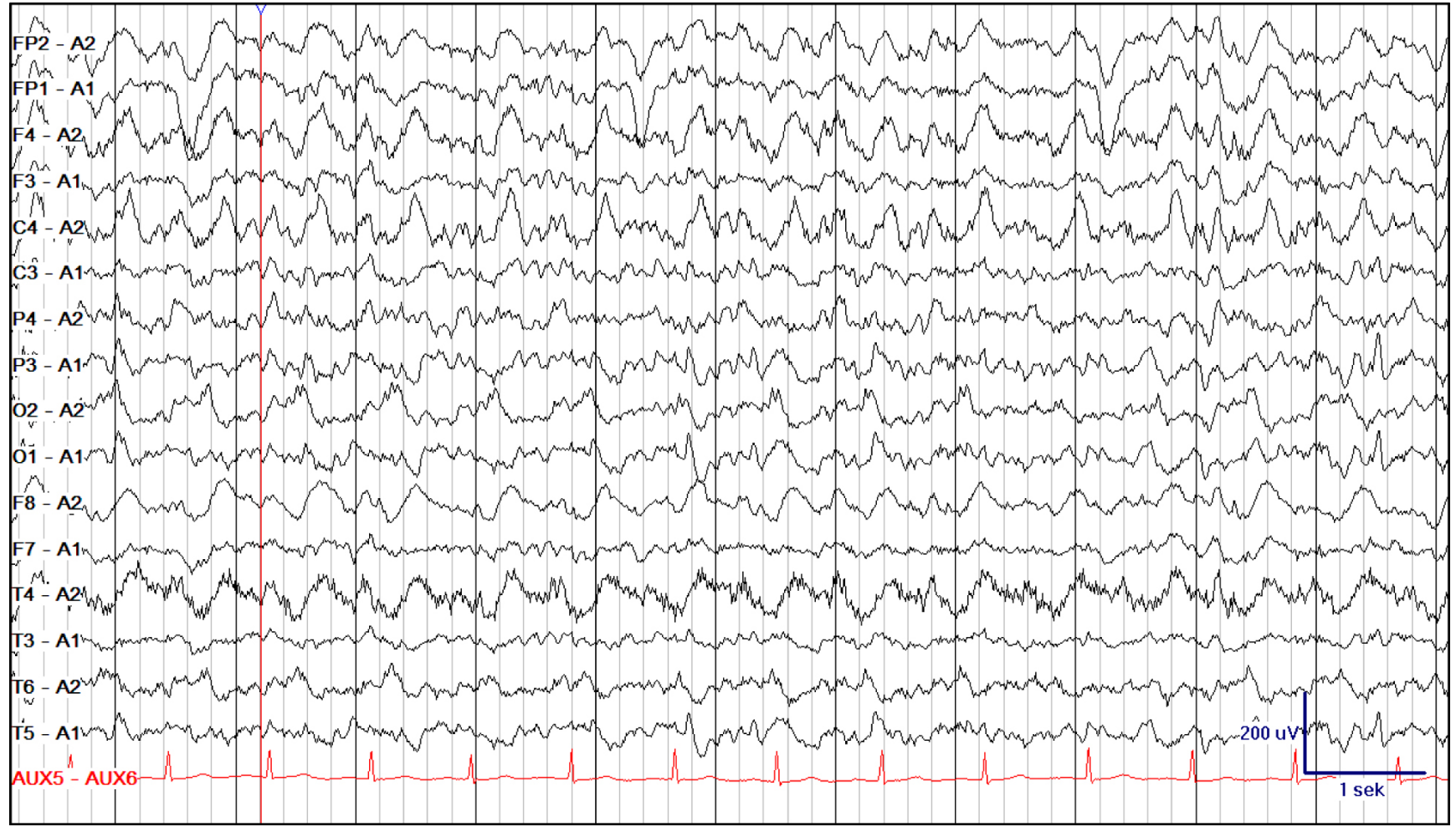

(b)

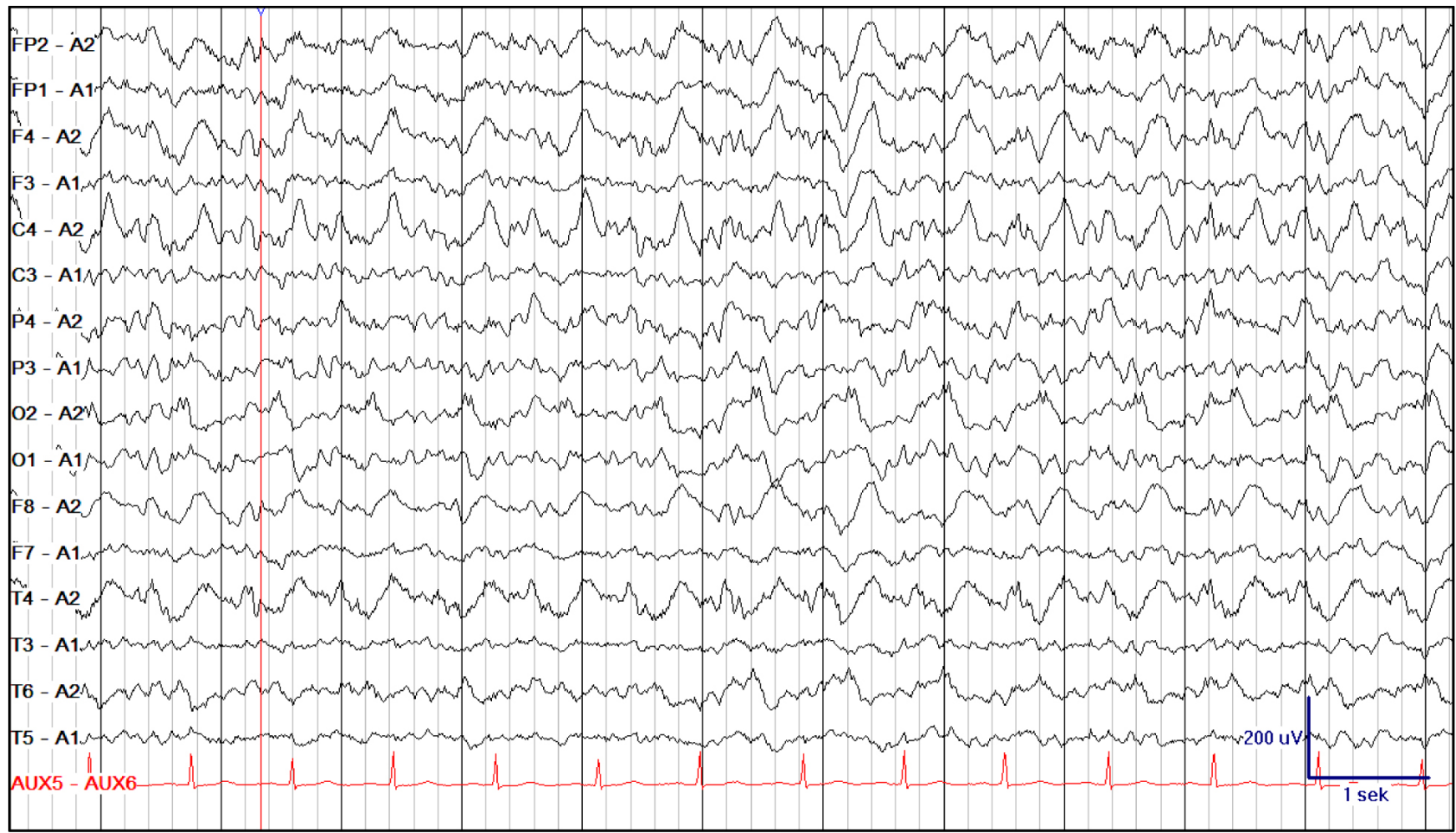

Fig. (2). EEG of a 79 year old female with a severe, temporary right hemispheric deficit after an epileptic seizure interpreted as a postictal (Todd) dysfunction. A single seizure and mental disturbance was caused by hypocalcaemia. Upper row: slow background activity with right sided PLEDS as periodic rhythmic sharp waves in frontocentrotemporal regions at approximately $1 \mathrm{~Hz}$. Note single independent sharp waves in left posterior regions (middle and end of EEG segment) Lower row: hardly any change in EEG activity after intravenous infusion of $2 \mathrm{mg}$ clonazepam, prompting the diagnosis of PLEDS rather than NCSE. She gradually recovered independent of resolution of PLEDS which persisted for more than two weeks. Note: clonazepam was given because of timelier availability. 
department or hospital. While a GCSE has to be treated as an emergency and with potentially aggressive medication there is uncertainty how to proceed in case of NCSE or focal SE. Experimental and clinical data suggest that there is tissue damage in GCSE but not in NCSE and therefore aggressive treatment of NCSE may not be justified [10, 30, 31].

\section{OUT-OF-HOSPITAL TREATMENT}

As overall treatment efficacy critically depends on early treatment [32] it might be wise to start treatment of GCSE out of hospital. This subject has been addressed in several studies which clearly could demonstrate that out of hospital treatment of generalized convulsive SE with benzodiazepines is efficient and save [33, 34]. In the largest study the use of intravenous diazepam (DZP), lorazepam (LZP), and placebo was compared in a double-blind prospective study design on more than 200 patients $[34,35]$. SE was terminated on admission to the hospital in $59.9 \%$ of those treated with LZP, $42.6 \%$ of those treated with DZP, and $21.1 \%$ of those treated with placebo. Surprisingly complications in terms of cardiac arrhythmia, need for respiratory support and hypotension, which are often attributed to the medication were less frequent after benzodiazepine $(10.6 \%$ and $10.3 \%$, respectively) as compared with placebo (22.5\%) It has to be stressed that in this study only one benzodiazepine was used for a single patient and the rate of complications may rise considerably after several different benzodiazepines were applied in an unsystematic manner. Naturally intravenous medication usually can only be given by professionals but there are also promising results possible with easy to handle benzodiazepine formulations. Most of this data up to now, has been evaluated for the out-of hospital treatment of acute seizures in children. Easiest to use and best results are achievable with nasal midazolam (MDZ) or lorazepam (LZP) while rectal administration of diazepam is efficient but often more difficult to handle [36]. Buccal MDZ is slower than nasal application and in an experimental setting needs higher doses to be effective [37]. Intranasal lorazepam exhibits approximately 4-fold duration of action when compared to that of intranasal midazolam and is therefore considered better for preventing seizure recurrence. While these alternative routes have not been clinically investigated in detail for SE in adults there are several studies in children and its use is generally recommended in this age group [36].

In case of professional out-of-hospital treatment usually intravenous DZP or MDZ is given as LZP needs to be stored refrigerated.

\section{IN-HOSPITAL TREATMENT}

First line therapy for GCSE are benzodiazepines like DZP, LZP, and MDZ [38, 39]. Recommendation of LZP as first choice treatment is firmly established: LZP as first choice as it has the smallest volume of distribution, the longest intracerebral half-life, is the least lipophilic and all in all has the best anticonvulsant effect [34]. Clinical studies demonstrated non significant but nevertheless relevant effects in favour of LZP with a probability for seizure termination of up to $64 \%[22,25,40]$. Usually separate boli of 2 or initially $4 \mathrm{mg}$ each are recommended, up to total dose of $0.1 \mathrm{mg} / \mathrm{kg}$. It is important to start treatment early and with adequate doses as there is convincing evidence that otherwise receptor changes worsen treatment responses and finally prohibit any significant benefit of gabaergic drugs [22, 32, 41, 42]. Benzodiazepines are approved in most countries for SE.

Alternative treatment can be initiated with either phenytoin (PHT), or its prodrug fosphenytoin which is not widely available but easier to use [43]. PHT is less potent than BZD but has the advantage not be sedating. It must be applied via a dedicated intravenous line as it interacts with other drugs and has a high risk of phlebitis and tissue damage, especially once injected extravasally. The latter is more likely to occur during $\mathrm{SE}$ due to the ongoing convulsions. Another disadvantage is the long infusion time compared to the use of BZD. In up to $1,5 \%$ of patients treated with intravenous PTH a purple glove syndrome can evolve [44]. Cardiac side effects in terms of arrhythmias have been reported and care must be taken in patients with known heart-disease. Most often PHT is recommended after treatment was initiated with BZD and SE persists for longer than 20 minutes [25]. This can be considered a refractory SE and its treatment is challenging and has not been properly evaluated in controlled and prospective studies $[12,16,45$, 46].

The proposed dose of PHT in treatment of SE is $15-20$ $\mathrm{mg} / \mathrm{kg}$ body weight given intravenously with a maximum infusion speed of $50 \mathrm{mg} / \mathrm{min}$ thus for a total time period of approximately 30 minutes in a $75 \mathrm{~kg}$ adult.

A further early alternative is the use of i.v. valproic acid (VPA) which is not licenced as first line therapy but can be used in the course of an SE [47, 48]. The initial dose of VPA should be about $20-30 \mathrm{mg} / \mathrm{kg}$ bodyweight given as intravenous bolus, optionally followed by continuous infusion of $5-10 \mathrm{mg} / \mathrm{kg}$ body weight. There is insufficient data as to generally recommend VPA but all studies demonstrate little side effects even after high initial doses. Comparisons of VPA with either DZP or PHT could not show significant differences for seizure control $[49,50]$. A risk of VPA is the development of liver failure, pancreatitis and encephalopathy. The latter can be difficult to distinguish from ongoing SE and needs early diagnosis including EEG to prevent further, harmful infusion of VPA [51].

All further treatment should be performed by experienced staff and ideally on an ICU. At this stage anaesthetics are used. With the risk of intubation the risk of short-term mortality increases. A variety of anaesthetics (usually barbiturates, midazolam or propofol) have been used but because of a lack of prospective trials no general recommendation can be given [52]. In one metaanalysis non significant superiority of pentobarbital over propofol or MDZ could be demonstrated [46]. At this stage the EEG is usually used to adjust dosing and it is accepted to induce a "burst-suppression" pattern in the EEG (Fig. 4). The idea is to stop epileptic activity and to slow down brain metabolism to reduce neuronal damage [46]. There is, however, no consensus of how long the suppression period must be to have beneficial effects. It can be proposed to induce coma for at least 24 hours and try to reduce the dose under clinical and EEG monitoring $[25,52]$. All anaesthetics can induce severe hypotension and propofol can cause the potentially fatal "propofol infusion syndrome", especially when used 


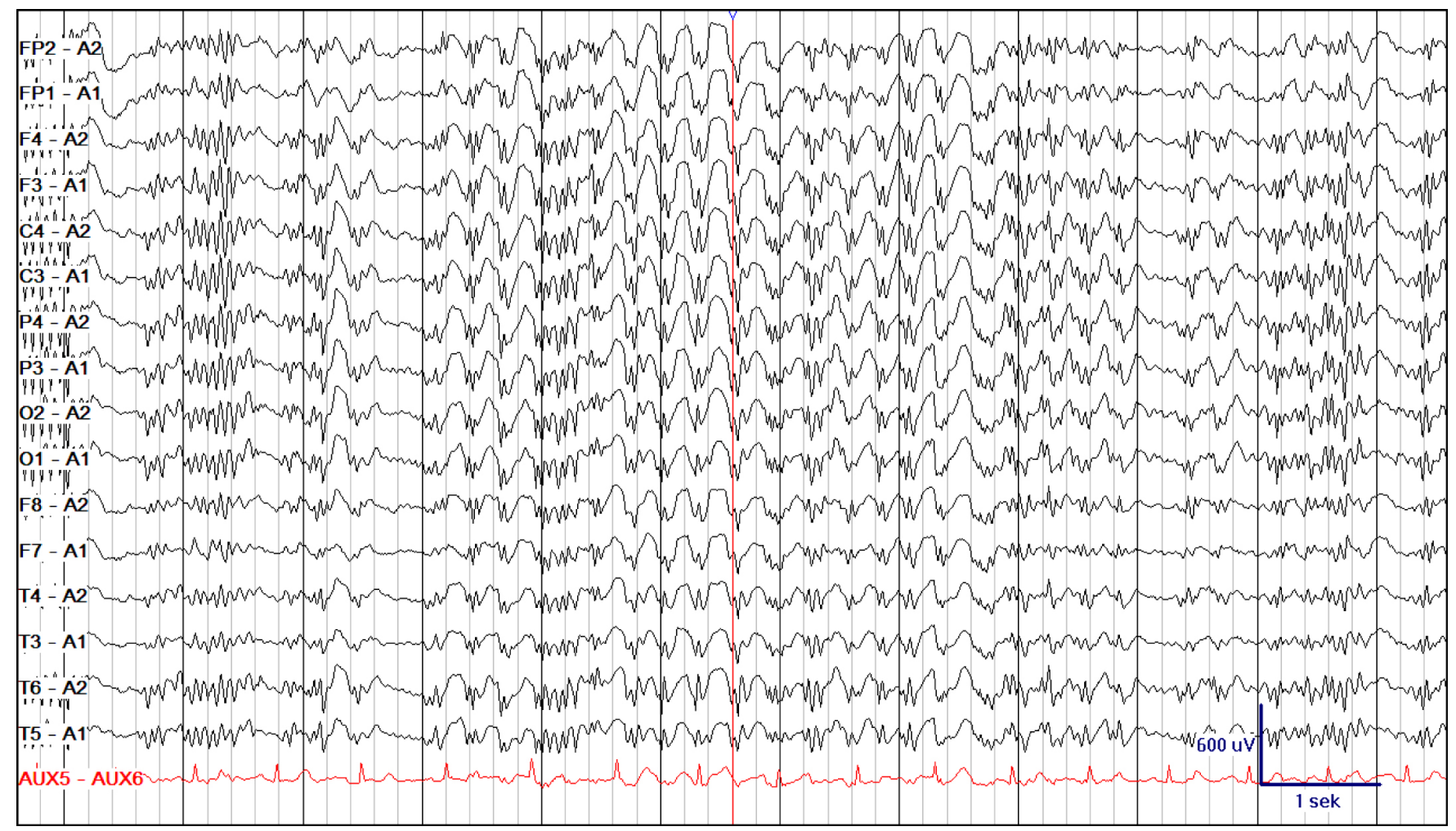

Fig. (3). EEG of a 46 year old female with late-onset absence status. She had a history of idiopathic generalized epilepsy with earlier history of absences some years ago, when tapering medication. The EEG depicts runs of generalized paroxysmal fast activity and irregular spike and wave and polyspike wave complexes with a frequency of $3 \mathrm{~Hz}$.

longer than a few days and in conjunction with catecholamines or steroids [53,54].

\section{FUTURE TREATMENT PERSPECTIVES}

On the basis that treatment of SE and refractory SE is difficult and can have deleterious side effects or even by itself worsen long-term prognosis after SE it is desirable to have potentially less harmful alternatives at hand. During the last years several modern anticonvulsants have been introduced which are highly potent, have little side effects, and partly are available as intravenous formulations. Especially levetiracetam, and lacosamide, which are available for intravenous administration, have been suggested for the treatment of SE, although none of them is licensed yet for this purpose $[6,55]$. Levetiracetam is well tolerated even with high dosing and infusion rates and several case reports and small series suggest promising results in the treatment of SE [56-60]. Infusion rates of up to $4000 \mathrm{mg}$ in 15 minutes or $2000 \mathrm{mg}$ in 5 minutes have been used without relevant side effects. Even less is known about the efficacy of lacosamide (LCM) as a treatment option in SE. Up to now there is only one report published with beneficial use of orally administered LCM in a patient with a refractory SE [61]. The efficacy in epilepsy, the pharmacological profile, and the tolerability, however, make it another promising treatment option in the future. Although both substances have the advantage of being available for consecutive oral use after status therapy, up to now they are not licensed and its use cannot be generally recommended. Probably, it can be advantageous to use these substances as an adjunctive therapy in refractory SE while sticking to established guidelines.

\section{SPECIAL CONSIDERATIONS IN NCSE}

The treatment of NCSE can be equally challenging as in GCSE but in general can be less aggressive $[10,31]$. Keeping in mind that a treatment regime designed for convulsive SE can induce coma and be followed by sequelae which may be worse than ongoing ictal neuronal activity, a prolonged and stepwise treatment protocol can be rectified. First line treatment, nevertheless, are benzodiazepines, which can also be of diagnostic value with a short diagnostic window during which the patient becomes neuropsychologically more adequate before sedation. Naturally, this treatment should be monitored with an EEG to allow the diagnosis. Higher doses of benzodiazepines and the use of anaesthetics should be avoided. In NCSE there are even more promising reports about the use of modern, potentially less harmful alternatives like valproic acid, levetiracetam, or lacosamide [30,62].

\section{PROGNOSIS}

Besides short term mortality rates there is increasing evidence for long term complications which hamper prognoses after treatment of GCSE [5, 15, 17]. Several studies have aimed to identify risk factors in order to tailor therapeutic measures early and recently a risk score was suggested [63]. Possible morbidity to follow can be neurological deficits, cognitive impairment and development of consecutive epilepsy in patients with de novo SE [17,64]. Factors influencing prognosis are age, refractory SE, and 


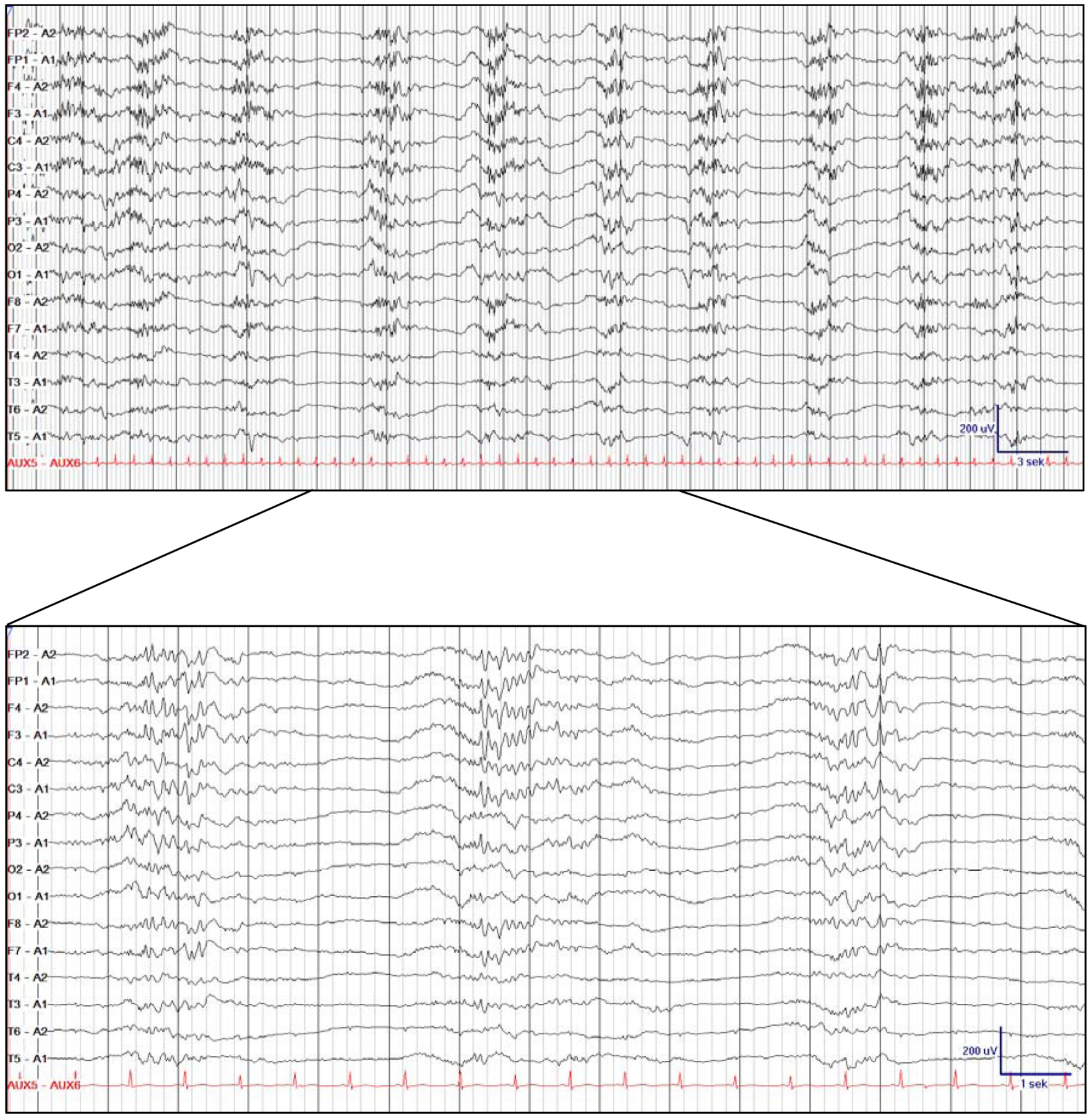

Fig. (4). Typical burst-suppression pattern of EEG after induction of coma by thiopental. Note different time scales in upper and lower part of figure.

aetiology of SE $[65,66]$. As has been mentioned earlier the worst prognosis follows refractory SE with the highest mortality, longer stays on the ICU and in hospital and a lower chance to regain cognitive baseline levels [17-19].

\section{CONCLUSION}

GCSE is an emergency which needs immediate treatment and has a high mortality. As early treatment is beneficial outof-hospital treatment can be advised. NCSE can only be diagnosed with an EEG and can be treated more carefully to prevent potentially harmful sequelae of therapy. The first- line treatment of GCSE is LZP which can be followed by PTH and induction of coma by anaesthetics. Some of the modern anticonvulsants may have good efficacy and tolerability but cannot be recommended yet. It is advisable to use an in-house protocol which all physicians are familiar with. Short-term prognosis to stop seizures become worse with time and the long-term prognosis is worst in case of refractory SE. Even after successful cessation of SE there is a high risk to develop epilepsy later on and not to recover to baseline level. 


\section{REFERENCES}

[1] Rosenow F, Hamer HM, Knake S. The epidemiology of convulsive and nonconvulsive status epilepticus. Epilepsia 2007; 48 Suppl 8: 82-84.

[2] DeLorenzo RJ, Pellock JM, Towne AR, Boggs JG. Epidemiology of status epilepticus. J Clin Neurophysiol 1995; 12: 316-25.

[3] Lowenstein DH, Alldredge BK. Status epilepticus. N Engl J Med 1998; 338: 970-76.

[4] Lowenstein DH, Bleck T, Macdonald RL. It's time to revise the definition of status epilepticus. Epilepsia 1999; 40: 120-22.

[5] Cooper AD, Britton JW, Rabinstein AA. Functional and cognitive outcome in prolonged refractory status epilepticus. Arch Neurol 2009; 66: 1505-09.

[6] Knake S, Hamer HM, Rosenow F. Status epilepticus: a critical review. Epilepsy Behav 2009; 15: 10-14.

[7] Rona S, Rosenow F, Arnold S, et al. A semiological classification of status epilepticus. Epileptic Disord 2005; 7: 5-12.

[8] Shorvon S. What is nonconvulsive status epilepticus, and what are its subtypes? Epilepsia 2007; 48 Suppl 8: 35-38.

[9] Coeytaux A, Jallon P, Galobardes B, Morabia A. Incidence of status epilepticus in French-speaking Switzerland: (EPISTAR). Neurology 2000; 55: 693-97.

[10] Meierkord H, Holtkamp M. Non-convulsive status epilepticus in adults: clinical forms and treatment. Lancet Neurol 2007; 6: 32939.

[11] Wu YW, Shek DW, Garcia PA, Zhao S, Johnston SC. Incidence and mortality of generalized convulsive status epilepticus in California. Neurology 2002; 58: 1070-76.

[12] Novy J, Logroscino G, Rossetti AO. Refractory status epilepticus: A prospective observational study. Epilepsia 2010; 51(2): 251-6.

[13] Proposal for revised clinical and electroencephalographic classification of epileptic seizures. From the Commission on Classification and Terminology of the International League Against Epilepsy. Epilepsia 1981; 22: 489-501.

[14] Jirsch J, Hirsch LJ. Nonconvulsive seizures: developing a rational approach to the diagnosis and management in the critically ill population. Clin Neurophysiol 2007; 118: 1660-70.

[15] Legriel S, Mourvillier B, Bele N, et al. Outcomes in 140 critically ill patients with status epilepticus. Intensive Care Med 2008; 34 : 476-80.

[16] Drislane FW, Lopez MR, Blum AS, Schomer DL. Detection and treatment of refractory status epilepticus in the intensive care unit. J Clin Neurophysiol 2008; 25: 181-86.

[17] Holtkamp M, Othman J, Buchheim K, Meierkord H. Predictors and prognosis of refractory status epilepticus treated in a neurological intensive care unit. J Neurol Neurosurg Psychiatry 2005; 76: 53439.

[18] Rossetti AO, Logroscino G, Bromfield EB. Refractory status epilepticus: effect of treatment aggressiveness on prognosis. Arch Neurol 2005; 62: 1698-1702.

[19] Mayer SA, Claassen J, Lokin J, Mendelsohn F, Dennis LJ, Fitzsimmons BF. Refractory status epilepticus: frequency, risk factors, and impact on outcome. Arch Neurol 2002; 59: 205-10.

[20] Claassen J. How I treat patients with EEG patterns on the ictalinterictal continuum in the neuro ICU. Neurocrit Care 2009; 11: 437-44.

[21] Bauer G, Trinka E. Nonconvulsive status epilepticus and coma. Epilepsia 2010; 51:177-90.

[22] Chen JW, Wasterlain CG. Status epilepticus: pathophysiology and management in adults. Lancet Neurol 2006; 5: 246-56.

[23] Treiman DM. Electroclinical features of status epilepticus. J Clin Neurophysiol 1995; 12: 343-62.

[24] Thomas P, Lebrun C, Chatel M. De novo absence status epilepticus as a benzodiazepine withdrawal syndrome. Epilepsia 1993; 34: 355-58.

[25] Meierkord H, Boon P, Engelsen B, et al. EFNS guideline on the management of status epilepticus in adults. Eur J Neurol 2010; 17(3): 348-55

[26] Singh RK, Stephens S, Berl MM, Chang T, Brown K, Vezina LG, Gaillard WD. Prospective study of new-onset seizures presenting as status epilepticus in childhood. Neurology 2010; 74: 636-42.

[27] Kalita J, Nair PP, Misra UK. A clinical, radiological and outcome study of status epilepticus from India. J Neurol; 257: 224-29.

[28] Kalviainen R. Clinical trials in status epilepticus-The clinical perspective. Epilepsia 2009; 50 Suppl 12: 75-76.
[29] Minicucci F, Muscas G, Perucca E, Capovilla G, Vigevano F, Tinuper P. Treatment of status epilepticus in adults: guidelines of the Italian League against Epilepsy. Epilepsia 2006; 47 Suppl 5: 915.

[30] Rupprecht S, Franke K, Fitzek S, Witte OW, Hagemann G. Levetiracetam as a treatment option in non-convulsive status epilepticus. Epilepsy Res 2007; 73: 238-44.

[31] Kaplan PW. No, some types of nonconvulsive status epilepticus cause little permanent neurologic sequelae (or: "the cure may be worse than the disease"). Neurophysiol Clin 2000; 30: 377-82.

[32] Wasterlain CG, Liu H, Naylor DE, et al. Molecular basis of selfsustaining seizures and pharmacoresistance during status epilepticus: The receptor trafficking hypothesis revisited. Epilepsia 2009; 50 Suppl 12: 16-18.

[33] Lowenstein DH, Cloyd J. Out-of-hospital treatment of status epilepticus and prolonged seizures. Epilepsia 2007; 48 Suppl 8: 9698.

[34] Alldredge BK, Gelb AM, Isaacs SM, et al. A comparison of lorazepam, diazepam, and placebo for the treatment of out-ofhospital status epilepticus. N Engl J Med 2001; 345: 631-37.

[35] Lowenstein DH, Alldredge BK, Allen F, et al. The prehospital treatment of status epilepticus (PHTSE) study: design and methodology. Control Clin Trials 2001; 22: 290-309.

[36] Sofou K, Kristjansdottir R, Papachatzakis NE, Ahmadzadeh A, Uvebrant P. Management of prolonged seizures and status epilepticus in childhood: a systematic review. J Child Neurol 2009; 24: 918-26.

[37] McDonough JH, Van Shura KE, LaMont JC, McMonagle JD, Shih TM. Comparison of the intramuscular, intranasal or sublingual routes of midazolam administration for the control of somaninduced seizures. Basic Clin Pharmacol Toxicol 2009; 104: 27-34.

[38] Leppik IE, Derivan AT, Homan RW, Walker J, Ramsay RE, Patrick B. Double-blind study of lorazepam and diazepam in status epilepticus. JAMA 1983; 249: 1452-54.

[39] Treiman DM, Meyers PD, Walton NY, et al. A comparison of four treatments for generalized convulsive status epilepticus. Veterans Affairs Status Epilepticus Cooperative Study Group. N Engl J Med 1998; 339: 792-98.

[40] Prasad K, Al-Roomi K, Krishnan PR, Sequeira R. Anticonvulsant therapy for status epilepticus. Cochrane Database Syst Rev 2005: CD003723.

[41] Chen JW, Naylor DE, Wasterlain CG. Advances in the pathophysiology of status epilepticus. Acta Neurol Scand 2007; 115: 7-15.

[42] Loscher W. Molecular mechanisms of drug resistance in status epilepticus. Epilepsia 2009; 50 Suppl 12: 19-21.

[43] DeToledo JC, Ramsay RE. Fosphenytoin and phenytoin in patients with status epilepticus: improved tolerability versus increased costs. Drug Safety 2000; 22: 459-66.

[44] Burneo JG, Anandan JV, Barkley GL. A prospective study of the incidence of the purple glove syndrome. Epilepsia 2001; 42: 115659.

[45] Abend NS, Dlugos DJ. Treatment of refractory status epilepticus: literature review and a proposed protocol. Pediatr Neurol 2008; 38: 377-90.

[46] Claassen J, Hirsch LJ, Emerson RG, Mayer SA. Treatment of refractory status epilepticus with pentobarbital, propofol, or midazolam: a systematic review. Epilepsia 2002; 43: 146-53.

[47] Kaplan PW. Intravenous valproate treatment of generalized nonconvulsive status epilepticus. Clin Electroencephalogr 1999; 30: $1-4$.

[48] Peters CN, Pohlmann-Eden B. Intravenous valproate as an innovative therapy in seizure emergency situations including status epilepticus--experience in 102 adult patients. Seizure 2005; 14: 164-69.

[49] Agarwal P, Kumar N, Chandra R, Gupta G, Antony AR, Garg N. Randomized study of intravenous valproate and phenytoin in status epilepticus. Seizure 2007; 16: 527-32.

[50] Misra UK, Kalita J, Patel R. Sodium valproate vs phenytoin in status epilepticus: a pilot study. Neurology 2006; 67: 340-42.

[51] Embacher N, Karner E, Wanschitz J, Beer R, Trinka E. Acute encephalopathy after intravenous administration of valproate in non-convulsive status epilepticus. Eur J Neurol 2006; 13: e5-6.

[52] Rossetti AO. Which anesthetic should be used in the treatment of refractory status epilepticus? Epilepsia 2007; 48 Suppl 8: 52-55. 
[53] Iyer VN, Hoel R, Rabinstein AA. Propofol infusion syndrome in patients with refractory status epilepticus: an 11-year clinical experience. Crit Care Med 2009; 37: 3024-30.

[54] Parviainen I, Kalviainen R, Ruokonen E. Propofol and barbiturates for the anesthesia of refractory convulsive status epilepticus: pros and cons. Neurol Res 2007; 29: 667-71.

[55] Perucca E. What is the promise of new antiepileptic drugs in status epilepticus? Focus on brivaracetam, carisbamate, lacosamide, NS1209, and topiramate. Epilepsia 2009; 50 Suppl 12: 49-50.

[56] Berning S, Boesebeck F, van Baalen A, Kellinghaus C. Intravenous levetiracetam as treatment for status epilepticus. J Neurol 2009; 256: $1634-42$

[57] Knake S, Gruener J, Hattemer K, et al. Intravenous levetiracetam in the treatment of benzodiazepine refractory status epilepticus. J Neurol Neurosurg Psychiatry 2008; 79: 588-89.

[58] Uges JW, van Huizen MD, Engelsman J, et al. Safety and pharmacokinetics of intravenous levetiracetam infusion as add-on in status epilepticus. Epilepsia 2009; 50: 415-21.

[59] Eue S, Grumbt M, Muller M, Schulze A. Two years of experience in the treatment of status epilepticus with intravenous levetiracetam. Epilepsy Behav 2009; 15: 467-69.
[60] Moddel G, Bunten S, Dobis C, et al. Intravenous levetiracetam: a new treatment alternative for refractory status epilepticus. J Neurol Neurosurg Psychiatry 2009; 80: 689-92.

[61] Tilz C, Resch R, Hofer T, Eggers C. Successful treatment for refractory convulsive status epilepticus by parenteral lacosamide. Epilepsia 2010; 51(2): 316-7.

[62] Kellinghaus C, Berning S, Besselmann M. Intravenous lacosamide as successful treatment for nonconvulsive status epilepticus after failure of first-line therapy. Epilepsy Behav 2009; 14: 429-31.

[63] Rossetti AO, Logroscino G, Milligan TA, Michaelides C, Ruffieux C, Bromfield EB. Status Epilepticus Severity Score (STESS): tool to orient early treatment strategy. J Neurol 2008; 255: 1561-66.

[64] Meierkord H. The risk of epilepsy after status epilepticus in children and adults. Epilepsia 2007; 48 Suppl 8: 94-5.

[65] Rossetti AO, Hurwitz S, Logroscino G, Bromfield EB. Prognosis of status epilepticus: role of aetiology, age, and consciousness impairment at presentation. J Neurol Neurosurg Psychiatry 2006; 77: 611-15.

[66] Tsai MH, Chuang YC, Chang HW, et al. Factors predictive of outcome in patients with de novo status epilepticus. QJM 2009; 102: $57-62$.

(C) Hagemann et al.; Licensee Bentham Open.

This is an open access article licensed under the terms of the Creative Commons Attribution Non-Commercial License (http://creativecommons.org/licenses/ by-nc/3.0/) which permits unrestricted, non-commercial use, distribution and reproduction in any medium, provided the work is properly cited. 